Case 1.-S. H. F., aged 48; machinist. From Indiana, one month in Colorado when first seen by me July 28 last. His lung disease is said to have commenced with a chill and fever May 1. $\mathrm{He}$ is not improving, I think because of tubercular pleurisy at base of right lung, the apex of which is excavated, and top of the other lung slightly affected. A case of laryngeal tuberculosis as well, with nasal complications, biljous temper ament and torpid liver. Not a favorable case by any means, when one considers that the daily ounce of purulent expectoration contained fifty-five tubercle bacilli "to the field" on September 15 , when the antiphth isic serum $T$. $R$. was first administered. The injections were given every alternate day below the shoulder blade commencing with $1 / 2$ c. c. and gradually increased until a decided reaction limit was found at $11 / \mathrm{ccc}$ Reaction temperatures inclined to be prolonged of $1 \frac{1}{2}$ to 3 degrees $\mathrm{F}$., were found after $1 \frac{1}{2}$ up to $17-10$ c.c. doses, and $I$ learned that I had a more powerful serum to contend with than I had ever used before. A return to 1 c.c. for the dose gave better results with no temperature rise above 99 for quite a while. Meanwhile up to October 30 , he had received 25 c.c. in twenty-one injections, the sputum had greatly diminished in quantity and showed "ten to field" in about two weoks and "one to field" in six weeks. The pleurisy had subsided, partly at least, controlled by wearing a restraining bandage devised to epecially limit the movement of the affected area. The effect was good and all the physical signs improved. Unfortunately this patient had to go back to his Indiana home, October 30 but he is still having the remedy administered there and reports improvement continued, with a gain in weight of twelv pounds since the commencement of the treatment.

Case 2.--F. J. M., aged 24; draftsman. Came to Colorado in May, 1897, from Massachusetts. Lung trouble probably dated back to pneumonia five years ago, and a severe cold one year ago. Decrease in weight of twenty-four pounds from his average to 108 pounds, both apices show an advanced fibrotuberculosis, the third stage being more marked on the right than on the left side. By this evidence the diagnosis of a non. active fibro-tuberculosis both sides was made. Serum T. $R$. has been given for its general effect with the result that we now have a diminution of expectoration and some improvement. The proportion of the very few bacilli in sputum not yet much changed, improvement in physical signs but no gain in weight, though bad environments and confined occupation, leading to frequent colds, are disturbing elements which have to be accounted for in this case.

Case 3.-Sept. 20, 1897. Mrs. H. Age 27. Came from Connecticut. Weight at this date ninety-nine pounds. Had a hacking cough for eleven years, and two years ago sympathetic whooping cough and fever at times. Now some restrained action at base of right lung where once she had pleurisy, but the chief trouble is a chronic catarrhal fibro tuberculosis, second stage, upper half of left lung, with softening spot threatening under left shoulder blade. Commenced the T. R. serum September 22 , and on alternate days gradually reached 1 c.c. for a dose which has not been exceeded. Bacilli to the field, seventeen large with streptococci and staphylococci. November 2 and 18, seven and eight to the field, small and beaded and lese "mixed" infection. November 28, the record contains this note-Has taken 27 c.c. antiphthisic serum and has now no reactions and physical signs have all improved. The temperature being below 99 degrees P.M. and weight now is 106 pounds.

Case 4.--J. A. R. Chemist, age 28, came from New Orleans, July 6,1897 . First seen by me September 18 . A case of tubercular broncho-fibrosis of six years standing with hemorrhagic tendency, first stage; both upper halves of the lungs, chiefly right. Up to date taken the T. $R$. serum treatment ten weeks every other day since September 19. The spirometrical record has improved from 170 cubic inches to 200 . He has improved in weight and in general as well as local signs. The record of his sputum examinations is as follows: September 18, 25.3 to the field ; September $30,20.3$ to the field ; October 18, 2.5 to the field. November 2 and 19 , one to five and one to ten fields. December 3, 4 to field (been confining himself too closely indoors.)

Case 5.-J. E. L. Age 24. Newspaper reporter. Came from Pennsylvania. July 4. A case of laryngeal tuberculosis with broncho-fibrosis originating some two years ago but aggravated by overwork at night, one year ago. First stage, left side chiefly affected, bui evidences of bronchial involvement top of both lungs. Has taken the serum treatment over six weeks (18 c.c.) with exercise, inhaling, etc.; doing well. Had left side pleurisy at one time which has disappeared. Expec. toration much lessened in quantity, chest sounds improved, weight increased six pounds. No elevation of temperature now, though there was a little during the first part of the treatment; voice which was quite hoarse has improved and strengthened.
Bacilli to field October 13, seven, November 20, one to three fields. December 14 none.

Case 6.-W. S. Book-keeper. Age 31. Arrived from Wisconsin, October 28. A case of tubercular broncho-fibrosis with suspicious laryngeal complication, both lungs affected in first stage, also has fistula in ano. Acclimatization may have something to do with his improvement for it has certainly been remarkable. Before giving the serum injection his temperature each day was from 98.5 degrees to 100.5 degrees and by the time 1 c.c. dose was reached the next day's rise was slightly increased. Since then 99.2 degrees has not been exceeded. December 3 , after 12 c.c. had been given, the record showed, weight increased 156 pounds, a gain of six pounds in one month. Fistula nearly stopped discharging, sputum lessened in quantity about five times, and the bacilli in it reduced from 23.2 to 2.8 to the field. The physical signs and general strength are also on the gain. In fact the outlook for this, as well as for the others of this group, is bright for a permanent arrest of the disease. There have been five or six others who have taken the same treatment for shorter periods, but not long enough for any definite results. One of them, however, shows great succeptibility and the diagnostic effect of the T.R. serum which is worthy of notice.

A gentleman aged 26, referred to me by his physician in Pennsylvania, was seen on October 22, having arrived in Denver two days previously. His trouble seemed to have originated ten months previously, and he had visited North Carolina without benefit. A laryngeal and pharyngeal tuberculosis with lung involvement very slight at both apices, with principal centering point in left inter-scapular region, seemed to me at my first examination to be all that there was to explain the case. But the effect of the T.R. serum injections made manifest the incompleteness of my search as well as that of several physicians who had examined him previously, namely, that I could not reach the 1 c.c. dose the general and fever reactions were so great and prolonged. At two times, after 7.10 and 6.10 c.c. injections, he informed me he had stayed in bed all day, he felt so ill and feverish, the grippy signs being similar to occasional reactions I have seen from the the old tuberculin. I felt confident there was a concealed cause somewhere. This the stethoscope finally revealed in the left infra-mammary region, where, over an area, not larger than a silver dollar, pleuritic friction with fine crackles were heard only at the end of forced inspiration, and without any pain. Under proper restraint, to promote adhesion and limit movement, I think this condition is gradually improving and that this case will also be a favorable one.

In closing I will add that this diagnostic power, a valuable evidence of the real efficiency of the new remedy, together with the infrequency of the resulting serum rash (though local reaction, infiltration, etc., at the point of needle puncture will sometimes be seen), is or ought to be a recommendation for this T. R. made serum; especially to those physicians who understand its import. That this preparation can be made standard and safe will also do much toward overcoming the natural prejudice which pertains to the use of either Koch's old or new tuberculin.

\section{HOW WE TREAT CONSUMPTIVES TODAY.}

Read before the Tri-State Medical Association of Alabama, Georgia and Tennessee, at Nashville, Tenn., Oct. 13, 1897.

BY PAUL PAQUIN, M.D.

$$
\text { st. Louis, Mo. }
$$

From the very beginning of the application of antitoxins in diphtheria, tetanus and other infections, it was hoped that it would also be beneficial in tuberculosis, and to that end many investigators have 
labored faithfully in all parts of the world. Maragliano, in Italy, first sought a remedy in the natural blood serum of the ass, and in the latter part of 1885 or in 1886, he reported the results of his work with the serum of the same animal, previously treated to increase immunization. Prior to this, however, Richet, Hericourt and others in Europe had used the natural $\mathrm{dog}$ blood serum in various forms of the disease. I began to immunize horses against this affection in 1894, and in January, 1895, reported the first clinical results with the first immunized horse blood serum ever obtained or used, so far as I am able to learn. Many criticisms, kind and unkind, and unwarranted reflections scarcely dignified, followed, and some have stooped to public persecution, but the work has progressed. Today, amidst the puerile denunciations of sciolists, I have the consolation of finding my humble researches respectfully considered by some of the leading scientific lights of Europe and America and, the results obtained with it, attested by conscientious clinicians of high reputation in many quarters of this and other countries.

In spite of the beneficial effects of climate and other forms of treatment in vogue, and the vaunted claims of recoveries said to have been produced by a hundred different processes, there seems to be no doubt that sero-therapy, being nature's own remedy in infections, is the most promising. None of the other therapeutic measures used seem to offer so much hope or confidence. A seventh of the population die from the effects of consumption in one form or another, and probably four-fifths of this percentage succumb to the disease affecting the respiratory organs. Such an excessive mortality due to one disease warranted the beginning and continuation of my experiments, and investigations by the whole profession, in any legitimate line, for the discovery of a treatment that shall subdue suffering, reduce the mortality and arrest the progress of inherited conditions of tuberculosis.

Tuberculosis may affect all the organs of the body, but its seat of preference is in the chest. The respiratory organs of this cavity offer, apparently, the best field for the germs to grow, and more beings succumb to pulmonary phthisis than to all the other forms of tuberculosis together. It is this form I shall consider chiefly in this paper. It is the kind we see every day.

First, what is the nature of pulmonary phthisis? It is, unfortunately, nearly always a complicated affection, even in the very early stages. Very early in the th development of the disease, we find associated with the bacilli of tuberculosis a number of other germs, among which some are as deadly and as destructive as the specific microbe. Comparatively speaking, tuberculosis is essentially a slow malady, even in acute cases. Its primary lesions consist of one or several local points of inflammation, due to the growth and multiplication of the bacilli, which occurs at the expense of the cells, their secretions and products, among which the germs are lodged. During this development, toxins are generated. They are poisonous, as the name indicates, and they cause the death of the cells around them and these, eventually, soften and become liquefied. It is thus that we obtain the first formation of caseous matter of pure tuberculosis which, in such conditions, is not mixed with the products or pus of the ordinary septic or pyogenic germs. During this first evolution of a case of tuberculosis, at its initial point of infection, a protective envelope is formed by nature's recuperative forces, and it is soon such as to surround the lesion, thus circumscribing it and limiting the pathologic development. Then we have formed a tubercle, which may vary in size from that of a point of a pin to a walnut or larger, the most voluminous nodules being usually the result of the junction of several small tubercles or their coalescence. If a section is made through tubercles at various stages of their development, it will be found that from a hard consistency at the beginning they gradually soften and, so far as germs are concerned, they first contain only the bacilli of tuberculosis. But, when softening of these tubercles takes place to the degree that their envelope ruptures, there is offered to the germs which are inhaled, a means of ingress into diseased tissues which are fit soil for the latter's development. This is the beginning of mixed infection. From that time on, the expectoration of a patient not only demonstrates the existence of consumption germs, but large numbers of other pathogenic microbes, such as the streptococcus, staphylococcus, the tetragenus and numerous other complicating organisms more or less effective in their power to destroy tissues. Such complications seem to be of tremendous power in the causation of softening of large areas of lung tissue and in the production of cavities. Then, we have to deal not only with the associated microbes and the lesions they produce, but also with the toxins which they generate and which, separately or collectively or, perhaps, in chemic combination, produce various forms of toxemia and alter the blood, the nervous system and, as a consequence, affects the whole organization banefully in a more or less pronounced manner.

Tuberculosis in the early stages, before serious microbic complications arise, produces as a rule a comparatively mild fever, the temperature seldom rising above 101 degrees $F$. When the complications appear, the temperature rises to 102 degrees F., 103 degrees $F$, and higher, according to the degree of infection, the extent of the lesions produced and the quantity and quality of the toxins. It is not necessary for the purposes of this paper, to go deeply into the pathology of tubercular developments. I merely desire to impress you with the importance of recognizing the nature of the lesions and other pathologic conditions, in a general and special way, before applying any remedy, and also the necessity of considering the peculiarities of each individual case, in order that the disease be not treated only on a general plan supposed to be applicable to all; but rather that each individual case be treated on its merits. It is not enough to treat consumptives as such. One should treat the patient against the general and special diseased conditions existing. The local lesions and the special effects thereof; the direct results of tubercular development upon remote organs or systems; the general depression dependent on such conditions; the individual characteristics and mode of life; the inherited predispositions; all these should be considered before instituting treatment. The disease yields or resists very differently, according to the nature of the infection, its effects and the peculiarities of the patient's individuality. It is needless to say, for instance. that patients who have inherited the elements that predispose to tuberculosis do not offer the same physical resistance to the destructive power of the germ of this disease as do those unfortunates who 
have contracted the malady by accident and have more vital energy. In concluding this preface to the discussion of the treatment of tuberculosis, I wish to say a word with reference to the method of producing the antitoxins which are most suitable for the treatment of consumption.

As stated above, my first publication on the subject of serum in tuberculosis was made early in 1895. At that time, I was not ready to publish in detail the modus operand $i$ of the laboratory processes. Since then $I$ have repeatedly, at various medical meetings and through the medical press, explained more or less fully the various methods of immunization which I have tested from time to time. In 1894, I realized that the simple use of toxins in the treatment of the horses used for the production of antitoxin was not sufficient to produce complete immunity because it had been demonstrated that not only the liquid toxin (viz., tuberculin) of the germ of consumption was poisonous, but also that the structure of the parasite contained poisonous elements of a deadly character. Thus it was, that very early in my work, I used not only tuberculin for injections of the animals used to produce antitoxic serum, but used besides the structure of dead germs or germs with vitality purposely lowered and then mixed with sterile water. I have used the live germs also, following injection of these solutions. Thus it will be seen, that at the beginning. I used chiefly tuberculin to immunize the horse, gradually adopting methods which insured, more and more, the effects of reaction upon both the liquid poisons and the germ substance. Some tuberculins I used at the outset were produced by recognized authorities; chiefly such as Koch and the Bureau of Animal Industry in Washington. By the mixture of these liquid toxins and the germs, seriously modified or not, all the elements of the tubercle bacillus entered into the immunizing agent. But a question of importance, with respect to the reaction of the animal to these injections arose during that work, and it was this: What proportion of the toxic element of the germs was absorbed by the economy of the horse? It was evident that the greatest quantity of the most virulent quality of all the tubercle toxins, be absorbed in order to produce the greatest reaction and the highest immunity. Koch of Berlin gave help to the production of more perfect immunizing material when he brought forth his new tuberculin, which he specifies under the name of T.R. This, according to the publications which have appeared, is nothing more than the poison in the structure of the germs of tuberculosis extracted by rubbing these germs in a mortar, and by centrifugalization means. For use, this preparation is mixed with sterile water or glycerin, or both, and may be preserved by the addition of a minute proportion of formaldehyde or .3 to .4 per cent. of trikresol (as is the case for the preservation of the serum itself). The object of this pulverization of the germ structure is to liberate the toxin as much as possible and facilitate the absorption and assimilation of every toxic principle they contain. We produced practically the same thing at the laboratories under my control, for at least two years, for the purpose of immunizing horses.

By injecting a mixture of tuberculin and pulverized germ substance into a horse, in doses varying from one cubic centimeter daily or periodically to thirty or more, during from two to four or six months, we get a blood with serum that has resisting powers more or less pronounced, against the development of tuberculosis.

Now, as to the treatment of the disease. Bearing in mind the essential factors that contribute to the pathology of tuberculosis and the various circumstances that may influence it, how should we treat the disease today? Is sero-therapy promising enough to warrant its use and warrant further experiments and investigations? Yes. If it arrests one case in a certain stage does it, therefore, promise to arrest another in a similar stage, irrespective of other influences favoring destruction? No. There is no one treatment applicable to all forms of tuberculosis, irrespective of the conditions complicating or influencing the malady, or irrespective of the actual pathologic conditions locally. Anti-tubercle serum can be justly expected to act only against the purely tuberculous processes, and this. by no other means than the usual physiologic interference which nature herself sets forth to neutralize the toxic products of infections and subdue the microbic invasions. Sero-therapy in the treatment of consumption has its reason of existence because it is based on natural law. Yet it should be sustained by such forms of special and general treatments as the conditions present may demand. This assistance is useful to facilitate and hasten the repair of the damage done to the organization. True, we have on record apparently complete recoveries from serious conditions, which have occurred after the exclusive use of anti-tubercle serum or serums for mixed infection; but we have a greater number of such recoveries and more numerous improvements as the result of scientific management of the patients and a properly combined treatment including serum as special medication. A patient may recover by the effect of serum alone, when the result of the disease is not such as to affect organs or systems of paramount importance, such as the lungs, the throat, the circulation, the nerves, the stomach, etc., so severely, as to preclude the possibility of their restoration by nature's own force, after the baneful effects of germs and their toxins have been removed by the antitoxin. If such organs or apparatus are diseased practically beyond repair, particularly when mixed infection exists to a serious degree, anti-tubercle serum, if it does arrest the progress of the trubercle bacilli, can not directly repair the physical and physiologic loss of the tissues and their vitality, and death may ensue. Here is where accessory treatments are needed. If there be a mixed infection and serum is tried, a serum prepared specially for this condition is essential, such as the anti-streptococcus serum, which is produced on the same general principle as that for tuberculosis or diphtheria, and which is applicable in many of the usual mixed infections of any disense.

The aim of sero-therapy in tuberculosis then is, first of all, to counteract the effect of the toxin; second, to interfere with the progress of germ life; third, to restore or create anew in the organization physiologic recuperative powers, thus assisting in restoring the equilibrium of the faculties and the vitality of the organization as a whole. It is plain, therefore, that the serum treatment, like all others, promises most in the earliest stages.

From all that precedes in this paper, it would seem evident that in the treatment of consumption one should, first of all, pursue a wise course of management, and second, general and special treatments suitable to each case. Indeed, it may be stated as an 
axiom, that all the hygienic influences that may be brought to bear on the system to improve its vital powers, should be directed to play their part.

\section{GENERAL TREATMENT.}

Climate is a point which most practitioners justly insist on being properly considered. Dry climate, free from the sand and dust storms should be chosen, if practicable. It may be hot or cold; both are beneficial in different classes of patients. It should be remembered that some tuberculous patients suffer much from heat or cold, according to their condition, and others the reverse. As a rule, emaciated, anemic patients prefer a warm temperature and stand the offects of cold very poorly, while those with more flesh and warmer blood thrive more or less under its influence. Altitude is not of so much consequence. Palm Lake, in California, which is below the sea level and yet exceedingly dry, is said to be the most beneficial locality for consumptives, while equally good results are claimed for the altitudes from 2,000 to 10,000 feet as exist in Colorado, New Mexico, Texas and some states of Mexico. The beneficial effects of climate are said to depend chiefly on the germicidal and stimulating properties of ozone and the purity of the air; therefore, it may be obtained, in a measure, in a room in a damp climate, in which such air may be to a degree created, by means of electricity and otherwise.

As a rule, patients are removed to suitable climates when it is too late, when they have cavities and are almost unavoidably doomed. On the other hand, those who do go to such climates usually return home too soon. Indeed, those who migrate to dryer climes for lung diseases should remain there if it agrees with them. They should make it their home. To return from such places to dampness usually means relapse and death.

Hydrotherapy is sometimes useful in consumption, partioularly in the earlier stages, and more especially in those who have contracted the disease by contagion, without there being any inherited field for it. Then, salt rubs, all over the body, followed by a cool or cold douche or plunge of from one to five minutes duration, and then by a coarse towel friction, assist materially in stimulating the circulation and contribute to the well-being of the sufferer. These should be administered morning or evening.

Massage properly applied is another treatment of much utility, particularly in cases where there is indolence and a disposition to avoid exercise, even though the strength is sufficient to warrant it.

Dietetics are also of very great importance. Generally speaking the diet of consumptives should consist largely of animal albuminoids and heat producers of the most appetizing and nutritious character, the most easily digested and in the smallest bulk. While vegetable albumin is good, animal foods seem to be the most suitable, and among these tender beef, broiled, roasted or hoiled, stands, in the order named, preeminent. Lamb, chicken and game come next. Fresh fish is also good. Vegetables that are agreeable to the patient and do not disturb digestion should be allowed as part of the diet, but not in sufficient quantity to appease hunger or supplant meats. So it is with sugars and fruits, the latter being preferable. Milk, cream and butter are advisable, if well tolerated. In case of poor appetite, the whites of two to half a dozen eggs, one to three or four times a day, at meals or between meals, constitute an excellent diet, supplying as it does, a large amount of albumin in a small bulk, usually digestible even by an impaired stomach. These nay be given raw or in milk shakes or in the form of punches with a small amount of good sherry wine. Made dishes, fried articles of food, should be avoided. The excessive use of alcoholic stimulants is likewise contra-indicated, and so is tobacco.

\section{SPECIAL TREATMENT.}

First in line is the specific treatment; that form of therapeusis which aims to set interference in the way of microbic development. It may consist of prophylactic measures of the vaccinating order, or treatment by the action of immunizing and curative principles, bused on the laws underlying immunity and recovery from infections. As vaccinating agents, the various forms of tuberculin may be used. Being poisonous principles of the germ of the disease, their effect is limited to the production of a mild kind of tolerance of the consumption germ, by the system of the being inoculated. Consequently, it is only in the very earliest moment of suspicion of tuberculosis that one may expect a cure by the use of such a preparation. Antitubercle serum, on the contrary, being the product with which nature herself often cures tuberculosis without the guidance of the human hand, may be used in all stages with perfect propriety and safety and with the assurance of at least having done one's duty in the application of medicine. As said before, it is in the earliest stages that the disease can be more readily arrested, but being an antitoxin it is applicable in all stages and under all circumstances, except the very rare exceptions in which heart disease or other idiosyncrasies exist, which preclude the hypodermic injection of albuminoids or salty preparations, and sometimes even of water.

In cases of pure tuberculosis, anti-tubercle serum may be administered hypodermically, in doses ranging from 5 to $6 \mathrm{~ms}$. daily, 30 being the average maximum dose. The pluce of injection, when they are to be continued for some time, should be a locality where a heavy fold of the skin can be most easily lifted and the hypodermic needle plunged most deeply into the connecting tissue. I find the area in the back, towards the side, most suitable. See Fig. 1.

The untoward effects of serum injections are very uncommon, particularly if one is careful not to push injections too rapidly and in too large doses from the first. Occasionally, a local swelling occurs which may be reduced by the ordinary methods for such conditions. If precaution has been taken to disenfect the skin thoroughly at the point of injection, by washing the locality and then rubbing it with alcohol, or preferably formaldehyde, there is little chance of producing a swelling due to microbes, providing that the syringe and needle be thoroughly clean and free from germs of any kind. The kind of syringe that appears in Fig. 2 is most suitable. It is easily disinfected, is graduated with both systems; its needles are not so large as those of most instruments and therefore cause less pain. The serum itself is absolutely sterile. No one need fear infectious injury from that source, unless by serious carelessness germs are allowed to contaminate it.

Infrequently symptoms of arthritis develop, which 
resemble very much the ordinary symptoms of rheumatism and which subside after suspending injections for a few days, and under the treatment for rheumatism. The occurrence of urticaria and other skin eruptions is not uncommon, and usually avoidable by progressive doses.

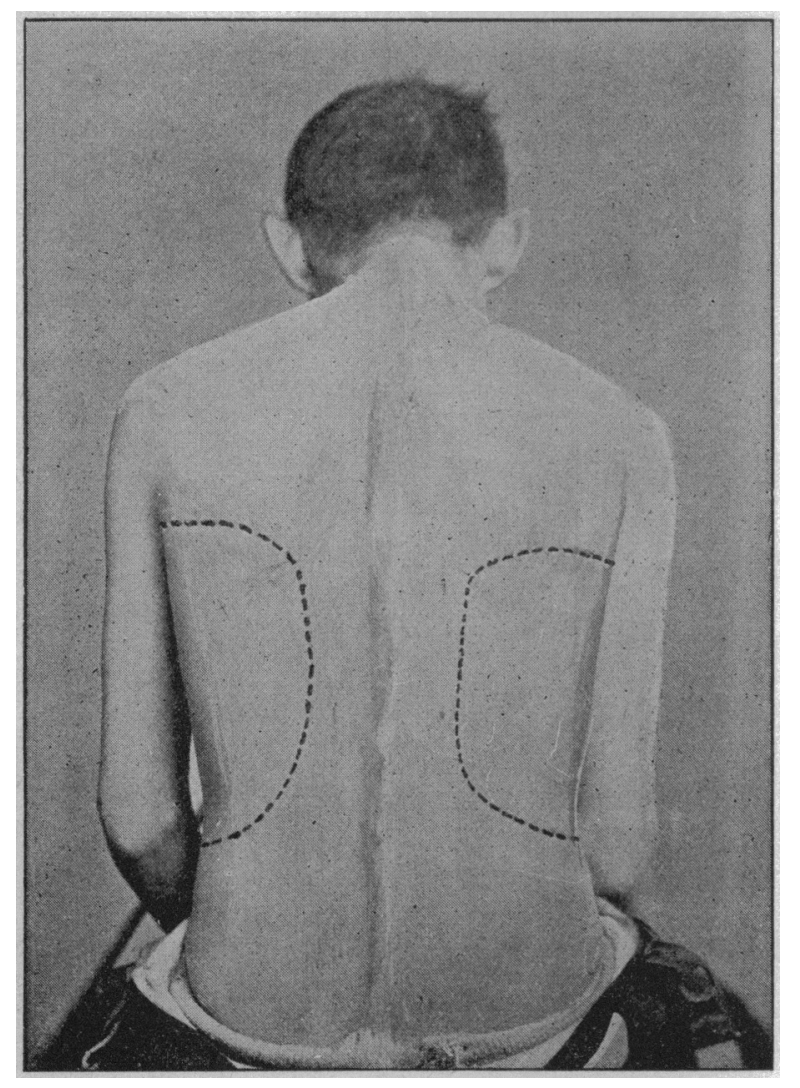

Figure 1.

The most striking, peculiar and perhaps most alarming of all the untoward symptoms of sero-therapy in any disease, is the strange and sudden interference with the circulation, manifested by dizziness, almost immediate cyanosis, and sometimes, more or less serious pain along the spinal cord, from the point of injection downward, including the nerve courses of the lower limbs, occasionally producing nausea and pain in the stomach. Sometimes these manifestations are followed by rigor. Fortunately, these results are ex. ceedingly rare and never have proved fatal. Over one hundred thousand injections of serum antitubercle (Paquin) are recorded without a serious result. In

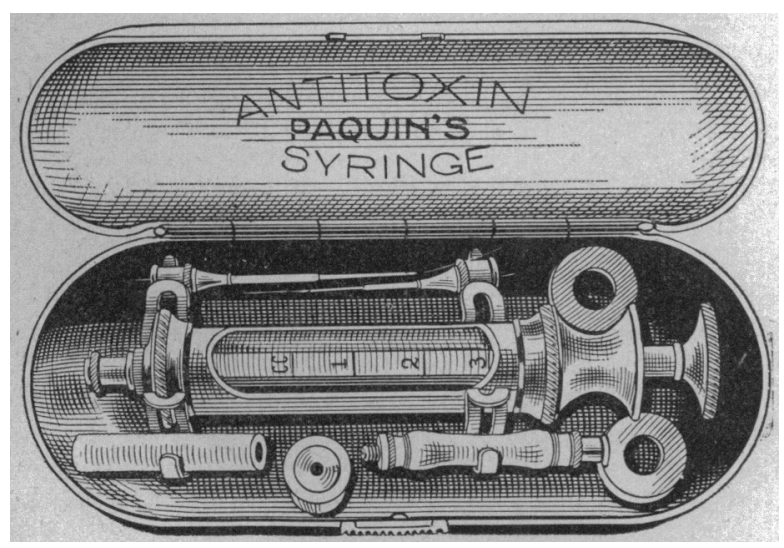

Figure 2. such cases, I usually discontinue hypodermic medication and use instead rectal injections with special syringe, Fig. 3, which gives as good results in many cases, although it requires usually a larger dose. As treatment of these effects, I prescribe nitrate of strychnia, hypodermically.

In mixed infections, particularly where the germs of pus predominate and where the expectoration is largely made up of pus and broken down tissue, I use the anti-streptococcus serum as accessory treatment. I produce this serum under the same principle that underlies the production of all other antitoxins, namely, by the immunization of the horse, by saturating injections of the toxins and germ substance of the streptococcus. This is done by the method known as Marmorek's, somewhat improved. This serum and the anti-tubercle serum may be mixed or given alternately on alternate days.

A.s to internal medication, it must depend wholly on the existing conditions. In anemia and other conditions suggesting feebleness of the nervous system and circulation, proper reconstructive blood tonics and nerve stimulants, such as pepto-mangan and nitrate of strychnia, are desirable. As to cough, unless it is very aggravating and prevents sleep to a serious degree, I never use opiates. I usually administer, when absolutely needed, codein and terpinhydrate.

It has been suggested that alteratives be used to assist in arresting the breaking down of tissue and glandular suppuration, etc., as is done in the treat. ment of syphilis. For that purpose, I have found a solution of iodin properly mixed with glycerin and some of the halogen salts, very beneficial. Iodin is credited, indeed, with having cured tuberculosis. The iodides are not good. They seem to destroy red blood corpuscles.

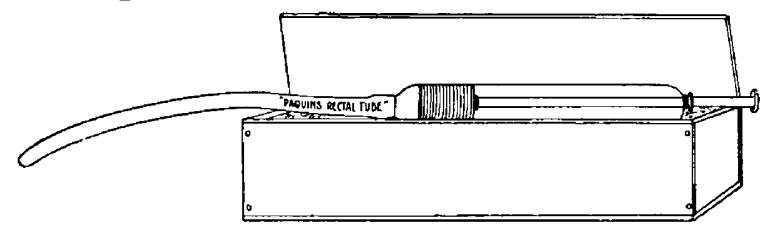

Figure 3.

As to inhalations, they should never be of an irritant character and, in fact, one should be very careful in their administration. In the early stages of tuberculosis most inhalations produce an irritation of the bronchi which is followed by a secretion that offers a good nidus for the development of germs. The inhaling ingredients containing the essence of pine needles, benzoin and such essences as are not irritant and are of a balsamic character, are most suitable. In cases with cavities, inhalations containing formaldehyde, are useful as disinfectants.

Electricity is also prescribed in tuberculosis. Both the galvanic and faradic currents have been used. The galvanic current is said to act by direct chemic action through the diseased organs, the poles being so placed as to pass the current through the affected parts. The benefits of this treatment are problematical, though sometimes they seem to be favorable. The faradic current is applied in a general way, as a stimulant of the general system.

Of recent date, so-called medicated electricity has been used in tuberculosis. It consists of treating the diseased portions by the application of the galvanic current with poles dipped in certain drugs. 
Iodin has been chiefly used for this purpose, and it is said that the current carries with it through the diseased organs principles which tend to arrest the progress of tuberculosis.

In closing, I wish to draw your attention to an exceedingly important point. It is, that most every physician expects too much of sero-therapy in tuberculosis. I have never made extravagant claims for it. It can not perform miracles and should be used early. It is neither just to the patient nor to the treatment to apply it as a last resort, when everything else has failed and the condition of the patient is such that doom stares him in the face.

\section{REPORT ON OXYTUBERCULIN IN THE TREATMENT OF TUBERCULOSIS.}

\section{BY J. O. HIRSCHFELDER, M.D.}

SAN FRANCISCO, CAL.

San Francisco, Nov. 20, 1897.

To the Editor:-I send you herewith a report on Dr. Hirsch. felder's oxytuberculin, together with his notes upon the cases examined, which we hope you will publish in full in the JourNAL. We are convinced of its efficacy and value, and urge its extended use. You will observe that it is not in any sense a secret nostrum, but has been placed legitimately before the profession. Yours truly, HENRY GiBBons, JR., M.D.

Since the address delivered before the California State Medical Society in April, 1897, upon "Oxytuberculin" the investigations described therein have been continued both in the bacteriologic and clinical departments.

The results that had been obtained up to that time have been confirmed by further observations under the scrutiny of many of my medical brethren.

The laboratory experiments proving the inhibitory action of the oxytuberculin upon the bacilli tubercuJosis have been repeated a great number of times with different modifications, but all teaching beyond doubt the certainty of the doctrine that oxytuberculin, even when greatly diluted with the culture medium most favorable for the germ, will prevent its growth. In all, forty-one series, of such culture experiments have been made by me and watched by my colleagues.

Continued treatment of many of the patients whose cases were reported at that meeting have shown that some of them have become entirely or nearly well, many have become much improved, while a fow have grown worse or have died. A table of all of these cases, as well as of such as have begun treatment since that time, will be herein reported.

Believing it the duty of Cooper Medical College to investigate the value of oxytuberculin, the faculty of that institution appointed a committee for that purpose, consisting of most of the professors of that institution.

October 24, after the committee had carefully inspected the laboratory evidence, the cases whose histories will be reported in detail in this paper were presented in person to this committee together with stained slides of the sputa prepared before, during and after the treatment. The cases were carefully investigated by the committee in every direction, physical examination of the chest and laryngoscopic examination of the throat being made where it was found necessary.

At the conclusion of this investigation at which a number of medical gentlemen not connected with the college were present and who participated in its deliberations, it was decided that the committee fully endorses the value of oxytuberculin, but desires to formulate its verbal approval in a lengthy report which shall be presented at the next faculty meeting, to become a matter of permanent record.

It was stated to the committee that I did not under. estimate the value of experiments conducted on animals, but that lack of facilities prevented me from conducting these in such a manner that their evidence, positive or negative, would be indubitable.

I stated that I considered it my duty to present all evidence collected, whether it be favorable or unfavorable, that it would not be proper to make a number of animal experiments and to select from these such only as gave favorable results, omitting others because the conditions under which they might have been performed were not such as to exclude accidents.

It has been my custom not to omit any observations that I have made bearing upon the subject, however unfavorable to my method the conditions may have been.

I stated that under the circumstances I deemed it wise not to enter upon the field of animal experiments, but to leave that department to those scientists who might be more favorably equipped for such researches.

The following are the cases that were presented to the committe:

Case 1.-F. A. A., merchant, aged 44 ; Jan. 15, $1896 . \quad$ No family history of phthisis. His wife died of tuberculosis last October, at which time he was subject to severe mental strain. For the past year he has been troubled with insomnia. For the past twenty years he has had occasional cough, with morning expectoration. Last October patient expectorated zss of pure blood. During the past six weeks the cough has been worse; he has lost eleven pounds in weight and has had one night sweat. The rectal temperature varies from 99.2 to 100.4 degrees. Examination showed patient to be a fairly well nour. ished, very pale individual. Head shows no peculiarity. Cervical glands are slightly enlarged; the thorax is long, broad, well arched and deep; the right side is slightly flatter than the left ; the right supraclavicular region is slightly sunken; both sides move equally in respiration; there is a slight dulness of the right apex, with faint bronchial respiration and a few râles; the area of cardiac dulness and heart tones is normal : the liver is 10 centimeters and spleen is not enlarged; the urine is normal, the spirometer test shows 3160-3230 c.c., and the hemoglobin 70 per cent. (Gowers' hemoglobinometer); the sputum contains large numbers of bacilli of tuberculosis; his weight is $1531 / 2 \mathrm{He}$ was injected with oxytuberculin in doses of $5 \mathrm{c.c}$ daily, and on January 30 , that is, two weeks after the treat. ment had been begun, he had already much improved, his appetite being better and the pationt feeling stronger. His cough was much less and the expectoration about one-third of what it had been before. His weight was 155 , the hemoglobin 90 per cent. ; at one examination no tubercular bacilli were found in the sputum. February 27 his weight had increased to 164 pounds and the spirometer showed $3260-3380$ c.c. At four examinations of the sputum during the month no bacilli tuberculosis were found, and on one occasion only a few. For fifteen days previous to February 29 no expectoration had occurred and the patient felt much stronger. In March the evening rectal temperature reached only to 99.6 , and on one occasion he coughed, raising a small quantity of phlegm, which contained a few bacilli. March 31 and April 20 no bacilli were found. May 23 he had a chill and the spleen was found to be enlarged. For this he was given quinin for a while, that being the only medicine he had received during the whole course of the treatment. June 4 was discharged cured, there having been neither cough nor expectoration since April 20 . The physical examination made by me and by Professor Kerr of the Medical Department of the University of California showed absolutely normal condition of the lung, no dulness whatsoever being present. The patient returned to his work, has gained further in weight and at the present time feels and looks perfectly well, and has remained so continuously since he was discharged from treatment on A pril 20, 1895. His present weight is 174 pounds.

Case 2.-Mrs. E. G., age 30, student. June 9, 1896. No family history of phthisis. Patient was well until two years ago when she had la grippe with bronchitis; since then she has been 